\title{
NuSTAR spectroscopy of GRS 1915+105: Disk reflection, spin, and connections to jets
}

Miller, J. M.; Parker, M. L.; Fuerst, F.; Bachetti, M.; Harrison, F. A.; Barret, D.; Boggs, S. E.; Chakrabarty, D.; Christensen, Finn Erland; Craig, W. W.

Total number of authors:

18

Published in:

The Astrophysical Journal Letters

Link to article, DOI:

10.1088/2041-8205/775/2/L45

Publication date:

2013

Document Version

Publisher's PDF, also known as Version of record

Link back to DTU Orbit

Citation (APA):

Miller, J. M., Parker, M. L., Fuerst, F., Bachetti, M., Harrison, F. A., Barret, D., Boggs, S. E., Chakrabarty, D., Christensen, F. E., Craig, W. W., Fabian, A. C., Grefenstette, B. W., Hailey, C. J., King, A. L., Stern, D. K.,

Tomsick, J. A., Walton, D. J., \& Zhang, W. W. (2013). NuSTAR spectroscopy of GRS 1915+105: Disk reflection, spin, and connections to jets. The Astrophysical Journal Letters, 775(2), [L45]. https://doi.org/10.1088/20418205/775/2/L45

\section{General rights}

Copyright and moral rights for the publications made accessible in the public portal are retained by the authors and/or other copyright owners and it is a condition of accessing publications that users recognise and abide by the legal requirements associated with these rights.

- Users may download and print one copy of any publication from the public portal for the purpose of private study or research.

- You may not further distribute the material or use it for any profit-making activity or commercial gain

- You may freely distribute the URL identifying the publication in the public portal 


\title{
NuSTAR SPECTROSCOPY OF GRS 1915+105: DISK REFLECTION, SPIN, AND CONNECTIONS TO JETS
}

\author{
J. M. Miller ${ }^{1}$, M. L. Parker ${ }^{2}$, F. Fuerst ${ }^{3}$, M. Bachetti ${ }^{4}, 5$, F. A. Harrison ${ }^{4}$, D. Barret ${ }^{4}$, S. E. Boggs $^{6}$, D. Chakrabarty $^{7}$, \\ F. E. Christensen ${ }^{8}$, W. W. Craig ${ }^{9,10}$, A. C. Fabian ${ }^{2}$, B. W. Grefenstette ${ }^{4}$, C. J. Hailey ${ }^{10}$, A. L. King ${ }^{1}$, D. K. Stern ${ }^{11}$, \\ J. A. Tomsick ${ }^{6}$, D. J. WALton ${ }^{3}$, AND W. W. Zhang ${ }^{12}$ \\ ${ }^{1}$ Department of Astronomy, The University of Michigan, 500 Church Street, Ann Arbor, MI 48109-1046, USA; jonmm@umich.edu \\ ${ }^{2}$ Institute of Astronomy, The University of Cambridge, Madingley Road, Cambridge CB3 OHA, UK \\ ${ }^{3}$ Cahill Center for Astronomy and Astrophysics, California Institute of Technology, Pasadena, CA, 91125, USA \\ ${ }^{4}$ Universite de Toulouse, UPS-OMP, F-31400 Toulouse, France \\ ${ }^{5}$ CNRS, Institut de Recherche en Astrophysique et Planetologie, 9 Av. Colonel Roche, BP 44346, F-31028, Toulouse Cedex 4, France \\ ${ }^{6}$ Space Sciences Laboratory, University of California, Berkeley, 7 Gauss Way, Berkeley, CA 94720-7450, USA \\ ${ }^{7}$ Kavli Institute for Astrophysics and Space Research, Massachusetts Institute of Technology, 70 Vassar Street, Cambridge, MA 02139, USA \\ ${ }^{8}$ Danish Technical University, DK-2800 Lyngby, Denmark \\ ${ }^{9}$ Lawrence Livermore National Laboratory, Livermore, CA, USA \\ ${ }^{10}$ Columbia University, New York, NY 10027, USA \\ ${ }^{11}$ Jet Propulsion Laboratory, California Institute of Technology, 4800 Oak Grove Drive, Pasadena, CA 91109, USA \\ 12 NASA Goddard Space Flight Center, Greenbelt, MD 20771, USA \\ Received 2013 July 26; accepted 2013 August 16; published 2013 September 18
}

\begin{abstract}
We report on the results of spectral fits made to a NUSTAR observation of the black hole GRS $1915+105$ in a "plateau" state. This state is of special interest because it is similar to the "low/hard" state seen in other black holes, especially in that compact, steady jets are launched in this phase. The 3-79 keV bandpass of NuSTAR, and its ability to obtain moderate-resolution spectra free from distortions such as photon pile-up, are extremely well suited to studies of disk reflection in X-ray binaries. In only $15 \mathrm{ks}$ of net exposure, an extraordinarily sensitive spectrum of GRS 1915+105 was measured across the full bandpass. Ionized reflection from a disk around a rapidly spinning black hole is clearly required to fit the spectra; even hybrid Comptonization models including ionized reflection from a disk around a Schwarzschild black hole proved inadequate. A spin parameter of $a=0.98 \pm 0.01$ ( $1 \sigma$ statistical error) is measured via the best-fit model; low spins are ruled out at a high level of confidence. This result suggests that jets can be launched from a disk extending to the innermost stable circular orbit. A very steep inner disk emissivity profile is also measured, consistent with models of compact coronae above Kerr black holes. These results support an emerging association between the hard X-ray corona and the base of the relativistic jet.
\end{abstract}

Key words: accretion, accretion disks - black hole physics - gravitation - relativistic processes - X-rays: binaries

Online-only material: color figures

\section{INTRODUCTION}

Reflection of hard X-ray emission from a "corona" onto the accretion disk can measure black hole spin, and can also serve as a powerful probe of the geometry of black hole accretion flows. Disk reflection spectra excited near to black holes will bear the imprints of gravitational red-shifts and strong Doppler shifts (e.g., Fabian et al. 1989). As long as the accretion disk extends to the innermost stable circular orbit (ISCO; Bardeen et al. 1972), the degree of the distortions imposed by these shifts can be used to infer the spin of the black hole; efforts to exploit disk reflection as a spin diagnostic in X-ray binaries began in earnest over a decade ago. Owing to the fact that the effects on Fe $\mathrm{K}$ emission lines are especially pronounced features, and owing to the high flux levels observed in Galactic X-ray binaries, spin measurements have been made in a number of systems using this technique (e.g., Miller 2007; Miller et al. 2009).

In cases where the disk extends to the ISCO and the continuum is known to be fairly simple, not only can spin be inferred, the geometry of the corona can also be discerned. The best spectra and variability studies appear to point toward a very compact central corona ( $r \leqslant 10-20 G M / c^{2}$; e.g., Reis \& Miller 2013), consistent with prior results suggesting that hard $\mathrm{X}$-ray emission may arise in the base of a relativistic jet (e.g., Fender et al. 1999; Markoff et al. 2005; Miller et al. 2012). However, this is not yet clear, and it also unclear that this geometry holds universally.
Extremely high sensitivity - especially over a broad spectral band-provides a path forward in situations where the continuum and reflection spectrum may be more difficult to parse. NUSTAR detectors have a triggered read-out; unlike CCD spectrometers, they are not subject to pile-up distortions (Harrison et al. 2013). In this respect, NuSTAR is especially well suited to disk reflection studies of bright Galactic compact objects. Moreover, NUSTAR offers unprecedented sensitivity out to almost $80 \mathrm{keV}$, giving an excellent view of the Compton backscattering hump (typically peaking in the 20-30 keV), and any additional curvature or breaks.

GRS $1915+105$ is a particularly important source for understanding black hole spin, disk-jet connections in all accreting systems, and how accretion flows evolve with the mass accretion rate. Prior efforts to measure the spin of GRS 1915+105 have not come to a clear consensus. Moreover, a multiplicity of states are observed in GRS 1915+105 (Belloni et al. 2000). The most intriguing of these may be the so-called "plateau" state, because it bears the closest analogy with the "low/hard" state in other black hole transients. Notably, radio emission consistent with compact jet production and strong low-frequency quasiperiodic oscillations (QPOs) are observed in this state (e.g., Muno et al. 2001); when combined with sensitive spectroscopy, these features may offer unique insights into the inner accretion flow.

In Section 2, we describe the NuSTAR observation of GRS $1915+105$ and our reduction of the data. Section 3 


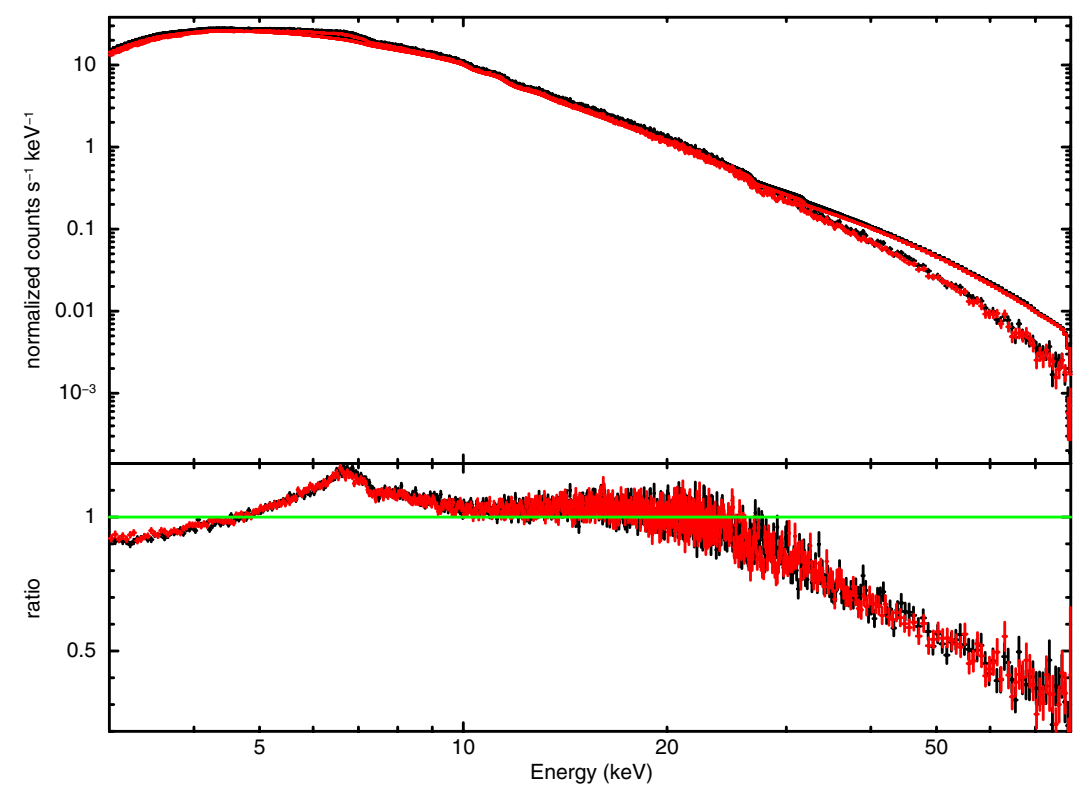

Figure 1. The 3-79 keV NUSTAR FPMA (black) and FPMB (red) spectra of GRS 1915+105, fit with a simple power-law assuming $N_{\mathrm{H}}=6 \times 10^{22} \mathrm{~cm}^{-2}$. The 4.0-8.0 keV and 15.0-45.0 keV bands were ignored in order to portray the curvature in the spectrum. A strong, skewed Fe $\mathrm{K}$ line is visible in the 4-8 keV band. The curvature in the $20-30 \mathrm{keV}$ band is due to a combination of a spectral cut-off and disk reflection.

(A color version of this figure is available in the online journal.)

describes our analysis of the FPMA and FPMB spectra. In Section 4, we discuss the results of our spectral fits and their impacts.

\section{OBSERVATIONS AND DATA REDUCTION}

NuSTAR observed GRS 1915+105 on 2012 July 3, over a span of $59.8 \mathrm{ks}$. The data were screened and processed using NuSTARDAS version 1.1.1. Spectra from the FPMA and FPMB detectors were extracted from $90^{\prime \prime}$ regions centered on the source position. Background spectra were extracted from regions of equivalent size on each detector; however, the background is negligible. Response files appropriate for the pointing (onaxis), source type (point, not extended) and region size were automatically created by the NuSTARDAS software. After all efficiencies and screening, the net exposure time for the resultant spectra was $14.7 \mathrm{ks}$ for the FPMA, and $15.2 \mathrm{ks}$ for the FPMB. The net observing time is small compared to the total observing due to the source flux, and in part because the observation occurred very early in the mission, and in part owing to detector dead-time.

The spectra were analyzed using XSPEC version 12.6 (Arnaud \& Dorman 2000). The $\chi^{2}$ statistic was used to assess the relative quality of different spectral models. We used "Churazov" weighting for all fits to govern the influence of bins with progressively less signal at high energy (Churazov et al. 1996). All errors reported in this work reflect the $1 \sigma$ confidence interval on a given parameter.

\section{ANALYSIS AND RESULTS}

Examination of the Swift/BAT light curve of GRS 1915+105 shows that our observation was made at the start of an $\sim 100$ day interval with sustained hard flux and only moderate variability. Intervals before and after have much stronger dayto-day variability. The light curve of our observation shows significant source variability on short time scales, typical of GRS $1915+105$, as well as moderately strong QPOs between
0.5-3.0 Hz. A full timing analysis will be reported in a separate paper (M. Bachetti et al. 2013, in preparation), but the fact of these variability properties helps us to make a secure identification of the source state. These timing properties, as well as the source flux observed by the Swift/BAT, are typical of the "plateau" state of GRS 1915+105 (e.g., Muno et al. 2001; Trudolyubov 2001; Fender \& Belloni 2004). Observations with the RATAN-600 radio telescope found that GRS 1915+105 varied between $12 \pm 3$ and $6 \pm 3 \mathrm{mJy}$ at $4.8 \mathrm{GHz}$ (S. Trushkin 2013, private communication) during the NuSTAR observation, consistent with relatively radio-faint "plateau" states.

Version 1.1.1 of the NuSTARDAS software and calibration has verified the detector response over the 3-79 $\mathrm{keV}$ band, partly through careful comparisons to the Crab. In all cases, the FPMA and FPMB spectra of GRS 1915+105 were jointly fit over the 3-79 keV band. An overall constant was allowed to float between the detectors to account for any mismatch in their absolute flux calibration; in all cases, the value of this constant was found to be 1.02 or less. In all fits, absorption in the ISM was fit using the "tbabs" model (Wilms et al. 2000), using corresponding abundances ("wilm") and cross sections ("vern"; Verner et al. 1996).

Figure 1 shows the FPMA and FPMB spectra of GRS $1915+105$, fit with a basic power-law model. The sensitivity of both spectra is excellent. Simple, broken, and cut-off power-law models all fail to fit the data. However, they approximate the continuum, and the prominence of the remaining disk reflection features in the spectra is readily discerned in the ratio plots in Figure 2. The power-law indices obtained in these simple fits are broadly consistent with values measured in fits to Suzaku spectra of GRS 1915+105 in the "plateau" state (Blum et al. 2009).

The "comptt" model describes thermal Comptonization (Titarchuk 1994). It also leaves strong reflection-like residuals, and does not provide an acceptable fit. The "nthcomp" model is essentially a more physical means of obtaining a cut-off powerlaw continuum by mixing thermal and non-thermal electron distributions (Zycki et al. 1999). Importantly, "nthcomp" is capable 


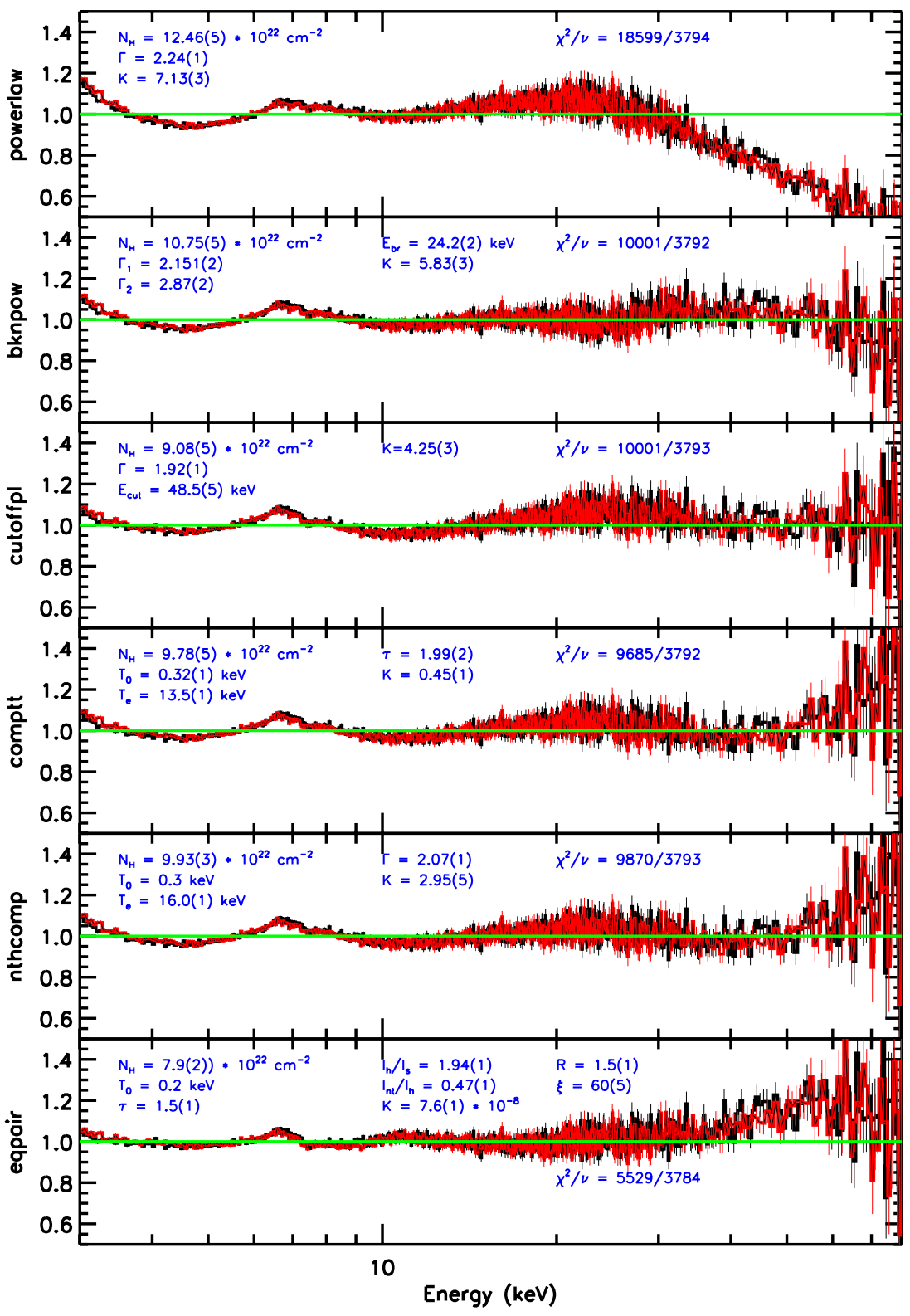

Figure 2. Data/model ratios obtained when the 3-79 keV FPMA (black) and FPMB (red) spectra of GRS 1915+105 are jointly fit with common spectral models. In each panel, the name of the spectral model is given on the vertical axis. The key parameters derived from each spectral fit, including the $\chi^{2}$ statistic, are given in each panel (in XSPEC parlance). In each case, "K" is the flux normalization of the model. Note that even "nthcomp" and "eqpair" (sophisticated Comptonization models) fail to describe the spectra owing to the strong, blurred reflection features that are present.

(A color version of this figure is available in the online journal.)

of accounting for curvature that might otherwise be mistaken for disk reflection. However, the data/model ratio and fit statistic in Figure 2 show that even "nthcomp" is unable to account for the strong, broad Fe $\mathrm{K}$ line and the Compton back-scattering hump.

The "eqpair" model describes Compton scattering in a sophisticated way, allowing mixtures of thermal and non-thermal electron distributions (Coppi 1999). "Eqpair" also explicitly includes blurred disk reflection. However, the reflection spectrum (described via the "pexriv" model; Magdziarz \& Zdziarski 1995) is blurred with the "rdblur" function (Fabian et al. 1989), which only describes the Schwarzschild metric and does not permit spin measurements. The internal reflection was coupled to an external "diskline" model, which is the kernel of "rdblur," in order to account for the emission line. In our fits, we fixed the cosine of the inclination angle to 0.3 , the elemental abun- dances to solar values, the inner disk radius to minimum possible $r_{\text {in }}=6 G M / c^{2}$, the outer radius to $r_{\text {out }}=1000 G M / c^{2}$, the emissivity to the Euclidian value of $q=3$ (recall that $J \propto r^{-q}$ ) and the disk temperature to $T=10^{6} \mathrm{~K}$ (the maximum allowed). The reflection fraction and disk ionization were allowed to vary. $\mathrm{Nu}-$ merous parameters control the hybrid thermal and non-thermal continuum. For simplicity, we fixed the disk blackbody temperature from which photons are up-scattered to $k T=0.2 \mathrm{keV}$, and varied the soft photon compactness $\left(l_{\mathrm{bb}}\right)$, the ratio of the hard to soft compactness $\left(l_{h} / l_{s}\right)$, the fraction of the power supplied to energetic particles that goes into accelerating non-thermal particles $\left(l_{\mathrm{nt}} / l_{h}\right)$, and the Thomson scattering depth $(\tau)$. The radius of the scattering region could not be constrained and was fixed at $1.5 \times 10^{6} \mathrm{~cm}$. Default values were assumed for all other parameters. Fitting "eqpair" in this way, a large improvement is achieved $\left(\chi^{2} / \nu=5529 / 3784\right.$; see Figure 2). 


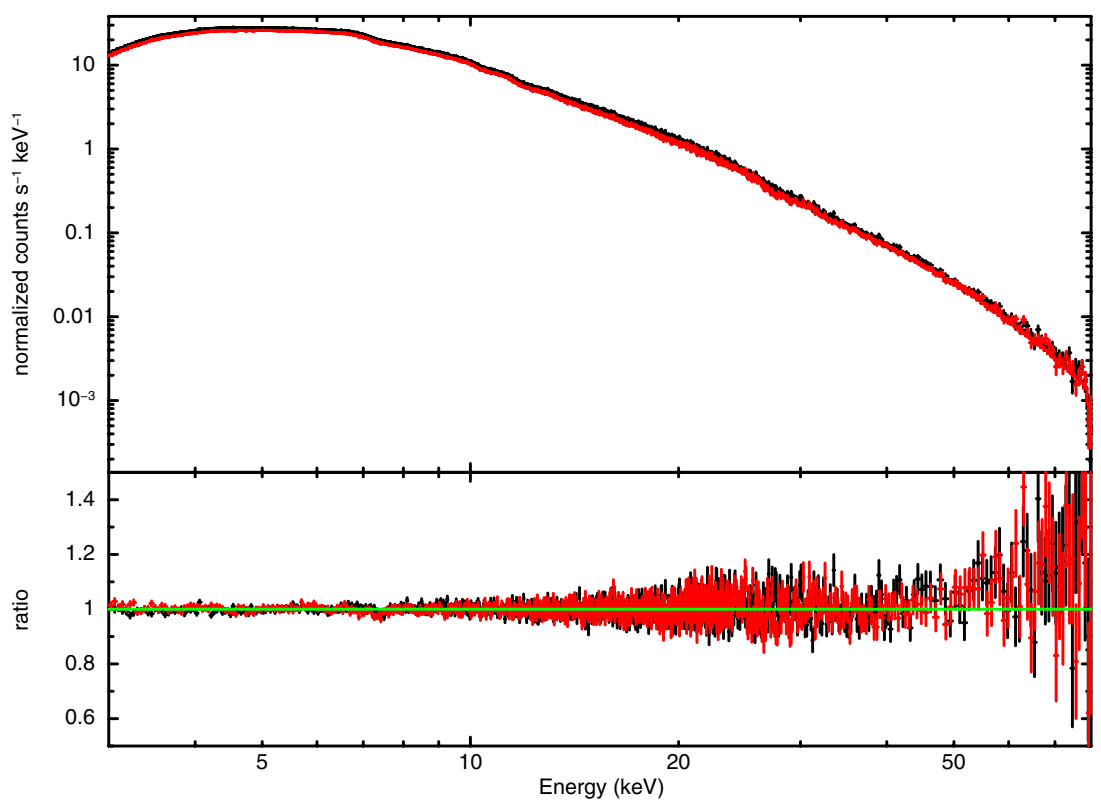

Figure 3. FPMA (black) and FPMB (red) spectra of GRS 1915+105, fit with a relativistically blurred disk reflection model. The continuum and reflection model include an exponential cut-off, as indicated by the simple fits, and consistent with prior results obtained in the "plateau" state. Using this model, a black hole spin parameter of $a=0.98$ (1) (statistical error only) is measured (see Table 1). The spectra were rebinned for visual clarity.

(A color version of this figure is available in the online journal.)

Table 1

Relativistically Blurred Disk Reflection Models

\begin{tabular}{|c|c|c|c|c|c|c|c|c|c|c|c|c|c|}
\hline Model & $\begin{array}{c}N_{\mathrm{H}} \\
\left(10^{22} \mathrm{~cm}^{-2}\right)\end{array}$ & $q_{\text {in }}$ & $q_{\text {out }}$ & $\begin{array}{c}r_{\text {break }} \\
\left(r_{g}\right)\end{array}$ & $\begin{array}{c}a \\
a \\
\left(c J / G M^{2}\right)\end{array}$ & $\begin{array}{c}\theta \\
(\mathrm{deg})\end{array}$ & $\Gamma$ & $\begin{array}{c}E_{\text {cut }} \\
(\mathrm{keV})\end{array}$ & $K_{\text {pow }}$ & $\begin{array}{c}\xi \\
\left(\mathrm{erg} \mathrm{cm} \mathrm{s}^{-1}\right)\end{array}$ & $A_{\mathrm{Fe}}$ & $\begin{array}{c}K_{\text {refl }} \\
\left(10^{-5}\right)\end{array}$ & $\chi^{2} / v$ \\
\hline Best-fit & $6.11(3)$ & $9.97(3)$ & $0.00^{0.01}$ & $6.5(1)$ & $0.983(3)$ & $72(1)$ & $1.720(2)$ & $35.6(3)$ & $2.59(1)$ & $1020(10)$ & $1.00(5)$ & $1.25(7)$ & $4070.6 / 3785$ \\
\hline$q_{\text {in }}=5$ & 6.26 & $5.0^{*}$ & 0.55 & 11.1 & 0.998 & 65.5 & 1.72 & 35.5 & 2.57 & 1230 & 1.0 & 0.86 & $4216.0 / 3786$ \\
\hline$r_{\text {break }}=3$ & 6.35 & 6.84 & 1.09 & $3.0^{*}$ & 0.977 & 71.9 & 1.74 & 35.6 & 2.59 & 1350 & 1.0 & 0.62 & $4260.9 / 3786$ \\
\hline Truncation & 6.17 & $3.0 *$ & $3.0 *$ & $6.0^{*}$ & $0.98 *$ & 65.0 & 1.74 & 36.0 & 2.52 & 1520 & 1.0 & 0.58 & $4547.4 / 3789$ \\
\hline$a=0$ & 6.44 & $3.0^{*}$ & $3.0^{*}$ & $6.0^{*}$ & $0.0^{*}$ & 65.0 & 1.77 & 40.0 & 2.10 & 5000 & 1.0 & 0.32 & $6113.2 / 3789$ \\
\hline
\end{tabular}

Notes. The parameters obtained for the best-fit relativistically blurred reflection model, tbabs $*$ kerrconv $*($ reflionx_hc + cutoffpl). The cut-off power-law normalization, $K_{\text {pow }}$, has units of photons $\mathrm{cm}^{-2} \mathrm{~s}^{-1} \mathrm{keV}^{-1}$ at $1 \mathrm{keV}$. Please see the text for additional details. The table also lists the results obtained for various models wherein parameters were fixed in order to explore the sensitivity of the fit statistic to plausible variations. Errors were only calculated for the best-fit model; the reported errors are $1 \sigma$ confidence limits. Parameters marked with an asterisk denote those fixed at a particular trial value in the rejected models. In the "truncation" model, the inner radius of the disk was fixed at $20 \times r_{\text {ISCO }}$.

Given these results, models focused on ionized disk reflection were next pursued. Our best-fit spectral model is constant $x$ tbabs $\times(($ kerrconv $\times$ reflionx_hc $)+$ cutoffpl $)($ see Table 1 , and Figures 3 and 4). "Kerrconv" is a relativistic blurring function, based on ray-tracing simulations (Brenneman \& Reynolds 2006). It includes inner and outer disk emissivity indices (following Wilkins \& Fabian 2012, $q_{1}$ floated freely but $q_{2} \geqslant 0$ was required), an emissivity break radius, the black hole spin parameter, the inner disk inclination (bounded between $65^{\circ}<i<80^{\circ}$, based on jet studies by Fender et al. 1999), and inner and outer disk radii (in units of the ISCO radius; values of $r_{\text {in }}=1.0$ and $r_{\text {out }}=400$ were frozen in all fits). "Reflionx_hc" is a new version of the well-known "reflionx" model that describes reflection from an ionized accretion disk of constant density (Ross \& Fabian 2005), assuming an incident power-law with a cut-off. These models capture important effects by solving the ionization balance within the disk, and scatter-broadening photoelectric absorption edges. That is, "reflionx_hc" includes broadening due to scattering, and this effect is balanced against dynamical and gravitational broadening when the model is convolved with "kerrconv." The power-law index of the hard emission in the "reflionx_hc" and "cutoffpl" models was linked in our fits, as was the characteristic exponential cut-off energy. The abundance of Fe within "reflionx_hc" was allowed to vary in the $1.0 \leqslant A_{\mathrm{Fe}} \leqslant 2.0$ range, and the ionization parameter was allowed to float freely $\left(\xi=L / n r^{2}\right)$. Flux normalizations for the "reflionx_hc" and "cutoffpl" models were also measured.

As shown in Table 1, the best blurred reflection model gives a fit statistic of $\chi^{2} / v=4070.6 / 3785$. This model returns a precise spin measurement: $a=0.98 \pm 0.01$. The quoted error is only the statistical error. Systematic errors are likely much larger, and related to the assumption that the optically thick disk truncates at the ISCO (see, e.g., Shafee et al. 2008; Reynolds \& Fabian 2008; Noble et al. 2010), and different methods and physics captured in different spectral models.

To obtain a broader view of the spin measurement and its uncertainty, we scanned the $0 \leqslant a \leqslant 0.998$ range using the "steppar" command in XSPEC. We made an initial scan with 


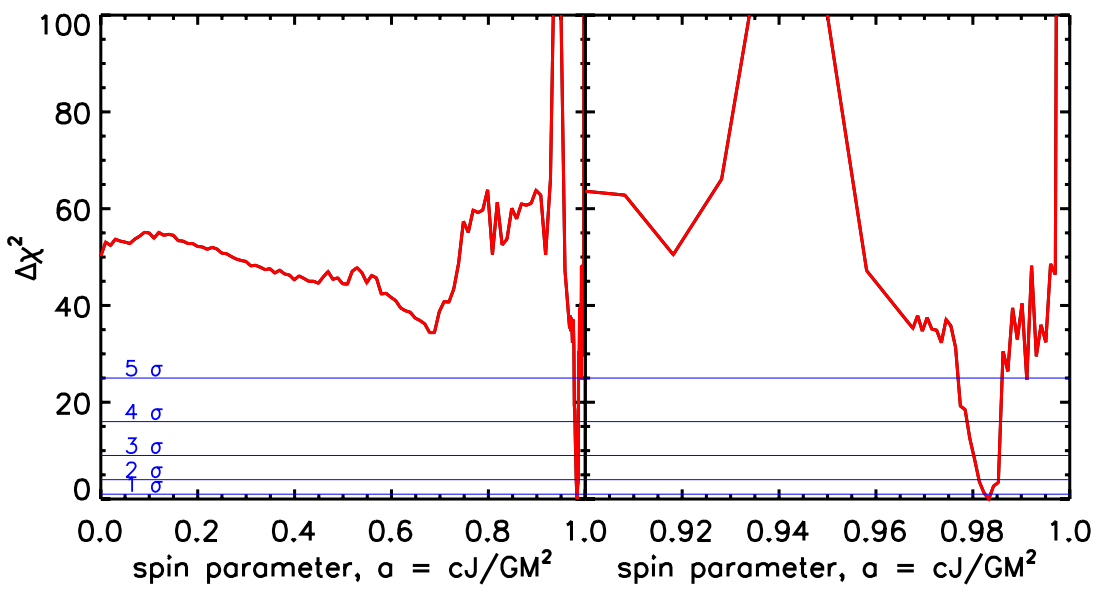

Figure 4. $\Delta \chi^{2}$ fitting statistic, plotted vs. different values of the black hole spin parameter $a=c J / G M^{2}$. The panel at left shows the full range, while the panel at right shows the $0.9 \leqslant a \leqslant 1.0$ range, for clarity. The spin measurement is based on relativistically blurred disk reflection modeling of the NuSTAR spectrum of GRS 1915+105 in the "plateau" state (see Table 1). The error range was scanned using the XSPEC tool "steppar," which allows all parameters to vary during the scan. The horizontal confidence levels indicate the Gaussian equivalent $\sigma$ value for the indicated change in $\chi^{2}$, assuming one interesting parameter.

(A color version of this figure is available in the online journal.)

100 points across the full band, and a second scan with 50 points in the $0.95 \leqslant a \leqslant 0.998$ range. Figure 4 shows the results of this error scan. There is a clear minimum at $a=0.98$; a maximal spin of $a=0.998$ is rejected at very high confidence, and so too are low spin values. It is notable that the $\chi^{2}$ versus $a$ contour shows local fluctuations, especially between $0.8 \leqslant a \leqslant 0.95$, although all $\chi^{2}$ values are significantly higher than achieved for the best-fit spin of $a=0.98(1)$. The fluctuations may indicate deficiencies in the spectral model, or could be partly due to the limited energy resolution of the spectra.

We also explored a number of fits with key parameters fixed at particular values (see Table 1). The data clearly prefer a solar abundance of $\mathrm{Fe}$, and the very steep inner emissivity index, for instance. The data strongly exclude a model with a much higher cut-off energy. As also indicated in Figure 4, the data rule out reflection from a black hole with zero spin at very high confidence (the model considered in Table 1 fixes $a=0$ and the emissivity indices at $q=3$, appropriate for a "lamp post" model in a Schwarzschild regime). Importantly, a plausible model for the low/hard state is also ruled out. The "truncated" model in Table 1 changed the best-fit model to require an inner disk radius fixed at 20 times the ISCO, and $q=3$. We also fit the "eqpair" model again, fixing $q=10$; this returned $\chi^{2} / v=$ $8059 / 3782$, potentially indicating the importance of spin effects.

The best-fit model in Table 1 gives a flux of $F=2.07(1) \times$ $10^{-8} \mathrm{erg} \mathrm{cm}^{-2} \mathrm{~s}^{-1}(0.1-100 \mathrm{keV})$. Adopting the mass and distance values favored by Steeghs et al. (2013), $M_{\mathrm{BH}}=10.1 \pm$ $0.6 M_{\odot}$ and $d=11 \mathrm{kpc}$, this flux gives a luminosity of $L=3.0(5) \times 10^{38} \mathrm{erg} \mathrm{s}^{-1}$ (where the error is based on an assumed distance uncertainty of $\Delta d= \pm 1 \mathrm{kpc}$ ), or an Eddington fraction of $\lambda=0.23 \pm 0.04$.

\section{DISCUSSION}

We have fit numerous models to an early broad-band $N u S T A R$ spectrum of GRS 1915+105, obtained in a "plateau" state. The sensitivity of the spectrum is extraordinary, in that the effects of continuum curvature and disk reflection can clearly be distinguished. Models that predict continuum curvature but which do not include reflection are unable to provide satisfactory fits. The data require a continuum with an exponential cut-off, and reflection from an ionized accretion disk around a black hole with a spin of $a=0.98(1)$.

Evidence of a relativistic disk line in GRS 1915+105 was first detected with BeppoSAX (Martocchia et al. 2002). Fits to the line detected in archival ASCA spectra recorded a steep emissivity and small inner radius $\left(r=1.8 r_{g}\right)$ commensurate with a spin approaching $a \simeq 0.9$ (Miller et al. 2005; similar values were subsequently found by McClintock et al. 2006 and Middleton et al. 2006 using the disk continuum). Two observations with XMM-Newton also detected broad lines but were inconclusive with respect to spin (Martocchia et al. 2006), as was a deep spectrum of GRS 1915+105 in the "plateau" obtained with Suzaku (Blum et al. 2009).

The measurement of a high spin parameter in a source known for jet production is interesting in that it may indicate that spin powers jet production, as predicted by, e.g., Blandford \& Znajek (1977). It is possible that the jet is powered partly by tapping the spin (Miller et al. 2009; Fender et al. 2010; Narayan \& McClintock 2012; Steiner et al. 2013; Russell et al. 2013). However, the broadest survey of available data suggests that the mass accretion rate and/or magnetic field may act as a kind of "throttle" (King et al. 2013a, 2013b) and do more to affect jet power.

The spectral fits presented in this paper also offer some potential insights into the geometry of the inner accretion flow, and into jet production. Compared to an Euclidean emissivity of $q=3$, the inner emissivity index is extremely steep ( $q \simeq 10$; see Table 1). This may ultimately be unphysical or incorrect; however, the same spin is obtained when $q=5$ is fixed (see Table 1). Our results appear to broadly confirm the predictions of independent ray-tracing studies that find steep and broken emissivity profiles for compact, on-axis, hard X-ray sources emitting close to rapidly spinning black holes (Wilkins \& Fabian 2011, 2012; Dauser et al. 2013). The emissivity is also predicted to flatten at moderate radii, again consistent with our results. Given that GRS 1915+105 launches compact radio jets in the "plateau" state (e.g., Muno et al. 2001; Trudolyubov 2001; Fender \& Belloni 2004), the hard X-ray region may plausibly associated with the base of the jet.

A very steep inner emissivity profile was recently reported in fits to the Suzaku spectrum of Cygnus X-1 in the "low/hard" state (Fabian et al. 2012). Joint Suzaku and radio monitoring of 
Cygnus X-1 in the "low/hard" state also concluded that the hard $\mathrm{X}$-ray continuum is likely produced in the base of the relativistic jet (Miller et al. 2012). More broadly, similar emissivity profiles have been seen in massive black holes accreting at relatively high Eddington fractions, notably 1H 0707-495 (Fabian et al. 2009). Studies of time lags in Seyferts and microlensing in quasars suggest that very compact coronae may be common (Reis \& Miller 2013).

Advection-dominated accretion flow models predict that the inner disk should be truncated at $\dot{m}_{\text {Edd }} \simeq 0.08$, or $\lambda \simeq 0.008$ (assuming an efficiency of 10\%; Esin et al. 1997). This is broadly consistent with the luminosity at which many sources transition into the "low/hard" state, wherein jet production is ubiquitous. Our results indicate that a steady jet can potentially be launched from a disk that extends to the ISCO. The disk, corona, and jet are undoubtedly a complex, coupled system, but jet production in black holes may be more closely tied to the nature of the corona than the inner disk radius. This may support a new model for jets and QPOs in accreting black holes (McKinney et al. 2012).

This NUSTAR observation has offered new insights into nature of the accretion flow in the "plateau" state, owing to its extraordinary sensitivity. Similarly, it has also provided the first strong spin constraint based on disk reflection modeling. However, additional modeling using developing disk reflection codes, and a deeper observation in the "plateau" state, are likely required in order to confirm these initial model-dependent results. A NuSTAR observation in a softer, more luminous state is likely also required in order to rigidly test and verify the spin measurement.

This work was supported under NASA contract No. NNG08FD60C, and made use of data from the NuSTAR mission, a project led by the California Institute of Technology, managed by the Jet Propulsion Laboratory, and funded by NASA. J.M.M. thanks Sergei Trushkin for communicating radio results.

\section{REFERENCES}

Arnaud, K. A., \& Dorman, B. 2000, XSPEC, https://heasarc.gsfc.nasa.gov/docs/ xanadu/xspec/

Bardeen, J. M., Press, W. H., \& Teukolsky, S. A. 1972, ApJ, 178, 347

Belloni, T., Klein-Wolt, M., Mendez, M., van der Klis, M., \& van Paraijs, J. 2000, A\&A, 355, 271
Blandford, R. D., \& Znajek, R. L. 1977, MNRAS, 179, 433

Blum, J. L., Miller, J. M., Fabian, A. C., et al. 2009, ApJ, 706, 60

Brenneman, L. W., \& Reynolds, C. S. 2006, ApJ, 652, 1028

Churazov, E., Gilfanov, M., Forman, W., \& Jones, C. 1996, ApJ, 471, 673

Coppi, P. 1999, in ASP Conf. Ser. 161, High Energy Processes in Accreting Black Holes, ed. J. Poutanen \& R. Svensson (San Francisco, CA: ASP), 375

Dauser, T., Garcia, J., Wilms, J., et al. 2013, MNRAS, 430, 1694

Esin, A. A., McClintock, J., \& Narayan, R. 1997, ApJ, 489, 865

Fabian, A. C., Rees, M. J., Stella, L., \& White, N. E. 1989, MNRAS, 238, 729

Fabian, A. C., Zoghbi, A., Ross, R. R., et al. 2009, Natur, 459, 540

Fabian, A. C., Wilkins, D. R., Miller, J. M., et al. 2012, MNRAS, 424, 217

Fender, R. P., \& Belloni, T. 2004, ARA\&A, 42, 317

Fender, R. P., Gallo, E., \& Russell, D. 2010, MNRAS, 406, 1425

Fender, R. P., Garrington, S. T., McKay, D., et al. 1999, MNRAS, 304, 865

Harrison, F. A., Craig, W. W., Christensen, F. E., et al. 2013, ApJ, 770, 103

King, A. L., Miller, J. M., Gultekin, K., et al. 2013a, ApJ, 771, 84

King, A. L., Miller, J. M., Raymond, J., et al. 2013b, ApJ, 762, 103

Magdziarz, P., \& Zdziarski, A. A. 1995, MNRAS, 273, 837

Markoff, S., Nowak, M. A., \& Wilms, J. 2005, ApJ, 635, 1203

Martocchia, A., Matt, G., Belloni, T., et al. 2006, A\&A, 448, 677

Martocchia, A., Matt, G., Karas, V., Belloni, T., \& Feroci, M. 2002, A\&A, 387,215

McClintock, J., Shafee, R., Narayan, R., et al. 2006, ApJ, 652, 518

McKinney, J. C., Tchekhovskoy, A., \& Blandford, R. D. 2012, Sci, 339, 49

Middleton, M., Done, C., Gierlinski, M., \& Davis, S. 2006, MNRAS, 373,1004

Miller, J. M. 2007, ARA\&A, 45, 441

Miller, J. M., Fabian, A. C., Nowak, M. A., \& Lewin, W. H. G. 2005, in the Proceedings of the Tenth Marcel Grossmann Meeting on General Relativity, Rio de Janiero, 2003 July 20-26, ed. M. Novell, S. Perez Bergliaffa, \& R. Ruffini (Singapore: World Scientific)

Miller, J. M., Pooley, G. G., Fabian, A. C., et al. 2012, ApJ, 757, 11

Miller, J. M., Reynolds, C. S., Fabian, A. C., Miniutti, G., \& Gallo, L. C. 2009, ApJ, 697, 900

Muno, M., Remillard, R., Morgan, E., et al. 2001, ApJ, 556, 515

Narayan, R., \& McClintock, J. 2012, MNRAS, 419, L69

Noble, S. C., Krolik, J. H., \& Hawley, J. F. 2010, ApJ, 711, 959

Reis, R. C., \& Miller, J. M. 2013, ApJL, 769, L7

Reynolds, C. S., \& Fabian, A. C. 2008, ApJ, 675, 1048

Ross, R. R., \& Fabian, A. C. 2005, MNRAS, 358, 211

Russell, D. M., Gallo, E., \& Fender, R. P. 2013, MNRAS, 431, 405

Shafee, R., McKinney, J. C., Narayan, R., et al. 2008, ApJL, 687, L25

Steeghs, D., McClintock, J., Parsons, S., et al. 2013, ApJ, 768, 185

Steiner, J., McClintock, J., \& Narayan, R. 2013, ApJ, 762, 104

Titarchuk, L. 1994, ApJ, 434, 570

Trudolyubov, S. P. 2001, ApJ, 558, 276

Verner, D. A., Ferland, G. J., Korista, K. T., \& Yakovlev, D. G. 1996, ApJ, 465,487

Wilkins, D., \& Fabian, A. C. 2011, MNRAS, 414, 1269

Wilkins, D., \& Fabian, A. C. 2012, MNRAS, 424, 1284

Wilms, J., Allen, A., \& McCray, R. 2000, ApJ, 542, 914

Zycki, P., Done, D., \& Smith, D. 1999, MNRAS, 309, 561 